\title{
Design of a Ka-band Propagation Terminal for Atmospheric Measurements in Polar Regions
}

\author{
Jacquelynne R. Houts, James A. Nessel, Michael J. Zemba \\ NASA Glenn Research Center, Cleveland, OH, USA
}

\begin{abstract}
This paper describes the design and performance of a Ka-Band beacon receiver developed at NASA Glenn Research Center (GRC) that will be installed alongside an existing Ka-Band Radiometer [2] located at the east end of the Svalbard Near Earth Network (NEN) complex. The goal of this experiment is to characterize rain fade attenuation to improve the performance of existing statistical rain attenuation models. The ground terminal developed by NASA GRC utilizes an FFTbased frequency estimation [3] receiver capable of characterizing total path attenuation effects due to gaseous absorption, clouds, rain, and scintillation by directly measuring the propagated signal from the satellite Thor 7.
\end{abstract}

Keywords - rain fade, radio wave propagation, propagation losses, radiometry, polar climate.

\section{INTRODUCTION}

As the current spectrum for satellite communications (SATCOM) systems is beginning to become increasing congested and the systems are reaching the limits of the capacity possible within the current allocations, the demand for higher frequency capability continues to rise. Current NASA SATCOM systems primarily utilize $\mathrm{X}$ and Ku-Band but plans to upgrade them to Ka-Band are underway [1]. However alongside the benefits of increased data rates and the departure from spectral crowding comes the escalation of atmospheric induced effects.

In order to statistically characterize the atmospheric effects on Ka-Band links NASA has conducted several propagation campaigns over the last decade. During the Advanced Communications Technology Satellite (ACTS) program five years of propagation data was collected in Alaska. The data collected from this campaigns has provided NASA with some insight as to the effect of the atmosphere at Ka-Band in Subarctic (High Latitude) Regions. However, the data collected in this regions has shown some deviations and/or inconsistent results with predictions from the global ITU-R models. To address this issue, several organizations around the world have begun conducting propagation experiments to characterize the atmospheric channel in the arctic.

The following sections will describe the design and laboratory measured performance of the Ka-Band beacon receiver that was built in-house by NASA Glenn Research Center (GRC). The design is based upon a Fast Fourier Transform (FFT) digital design that is used in other operational GRC propagation terminals [3-5]. This new design incorporates modifications that allow for the measurement of both the co and cross polarized signals with improved dynamic range.

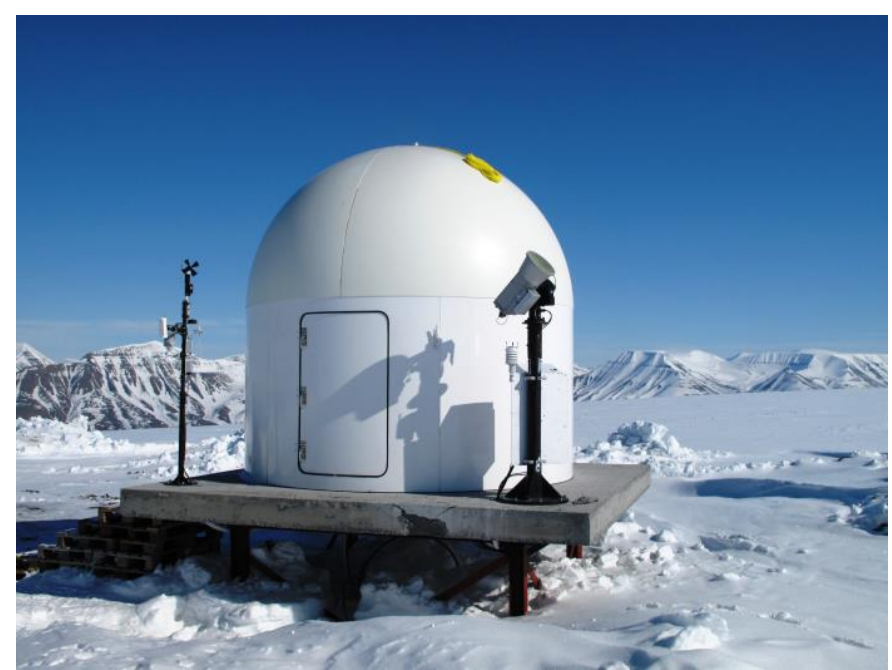

Figure 1: Photograph of the radome at the NEN site in Svalbard that will house the propagation terminal

\section{RECEIVER SPECIFICATIONS}

\section{A. Beacon Signal}

The propagation terminal will utilize the $20.198 \mathrm{GHz}$ beacon signal generated by the Telenor Sat THOR 7. THOR 7 is geostationary satellite with an EIRP of $19 \mathrm{dBW}$ located at $1^{\circ}$ West. Due to the position of the satellite the observation angle of the propagation terminal will be very near the horizon at an average of $2.65^{\circ}$. The horizon mask for THOR 7 from the ground terminal is shown in Figure 2.

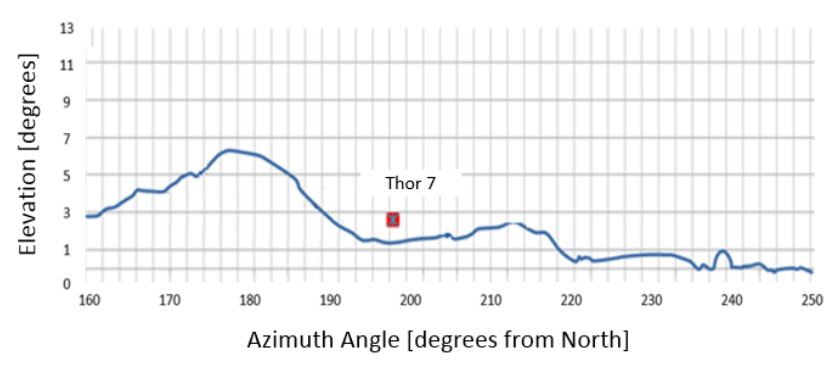

Figure 2: Horizon Mask of receive site

\section{B. Receiver Hardware}

The beacon receiver developed by NASA GRC consists of a $1.2 \mathrm{~m}$ K-band Cassegrain reflector antenna, followed by a polarizer and then an orthomode transducer which splits off the co and cross polarized signals. The antenna has a beamwidth of $.7^{\circ}$ which encompasses the orbital drift of the Thor 7 satellite 
without the need for tracking. The co and cross polarized signals are then passed through identical LNAs with $44 \mathrm{~dB}$ of gain before being down converted to the final intermediate frequency (IF) of $70 \mathrm{MHz}$. A block diagram of the front end and down conversion stage is provided in Figure 3.

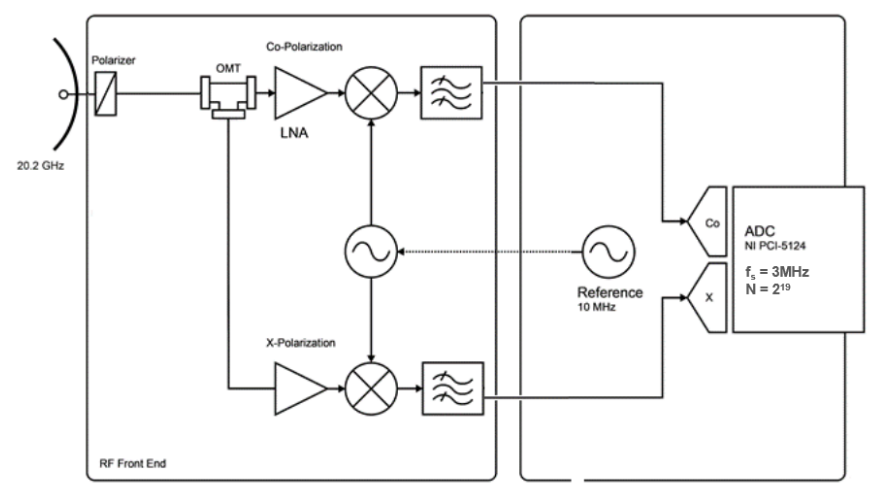

Figure 3: Block diagram of system

The co and cross polarized channels will both the transmitted via fiber to the data collection computer which will utilize the unique frequency estimation technique described in $[5]$.

The power level of both the co and cross polarized channel will be monitored and recorded at a rate of both $10 \mathrm{~Hz}$ and an average $1 \mathrm{~Hz}$. These measurements will be used for the characterization of atmospheric scintillation, depolarization and attenuation effects.

In order to ensure system stability the entire radio frequency $(\mathrm{RF})$ enclosure is temperature controlled and held at 40C $\pm 1 \mathrm{C}$. Additionally critical components are further temperature controlled and maintained within .01C of the setpoint.

Based on a preliminary link budget analysis and the parameters in the following table the dynamic range of the NASA GRC receiver is expected to be greater than $30 \mathrm{~dB}$.

Table 1: Receiver Specifications

\begin{tabular}{|c|c|}
\hline Parameter & Value \\
\hline System Noise Figure & $4.4 \mathrm{~dB}$ \\
\hline System Temperature & $720 \mathrm{~K}$ \\
\hline G/T & $17 \mathrm{~dB} / \mathrm{K}$ \\
\hline Sampling Rate & $10 \mathrm{~Hz} / 1 \mathrm{~Hz}$ \\
\hline XPD Isolation & $>30 \mathrm{~dB}$ \\
\hline
\end{tabular}

\section{Receiver Software}

The $70 \mathrm{MHz}$ IF signal is bandpass sampled with a sampling rate (fs) of $3.77 \mathrm{MHz}$. At a $10 \mathrm{~Hz}$ data measurement rate, 218 samples are collected for a final fs/ $\mathrm{N}$ resolution of $14.38 \mathrm{~Hz}$.

The receiver software is based upon an FFT digital design that is further augmented by the use of a novel frequency estimation technique. This technique utilizes a modified version of the Quinn-Fernandes frequency estimation algorithm that was primed with a priori information - a window in which the frequency is known or expected to appear, giving the algorithm a smaller search space. This algorithm and its accuracy are further discussed and documented in [5].

\section{RECEIVER PREFOREMANCE}

Before deployment in the field the propagation terminal underwent extensive testing in the laboratory. The system noise temperature of the receiver was measured to be approximately $720 \mathrm{~K}$. Based on the expected beacon signal levels from the THOR 7 payload (EIRP of 19dBW) and propagation path loss, a link budget was developed and indicates a clear sky receive carrier-to-noise density (C/N0) of $44 \mathrm{~dB}-\mathrm{Hz}$, with a $10 \mathrm{~Hz}$ measurement bandwidth the final clear sky carrier-to-noise ratio should be approximately $34 \mathrm{~dB}$.

Figures 4 and 5 show the system performance in simulated clear sky conditions in the laboratory. The figures show the 1min RMS time series recorded (calculated from the $10 \mathrm{~Hz}$ measurements) of the co and cross polarized channel over 24 hours in the laboratory. From these plots it can be observed that the measurement resolution during clear sky conditions is $.05 \mathrm{~dB}$ root-mean-square (RMS) for the co polarized signal and $.05 \mathrm{~dB}$ RMS for the cross polarized signal.

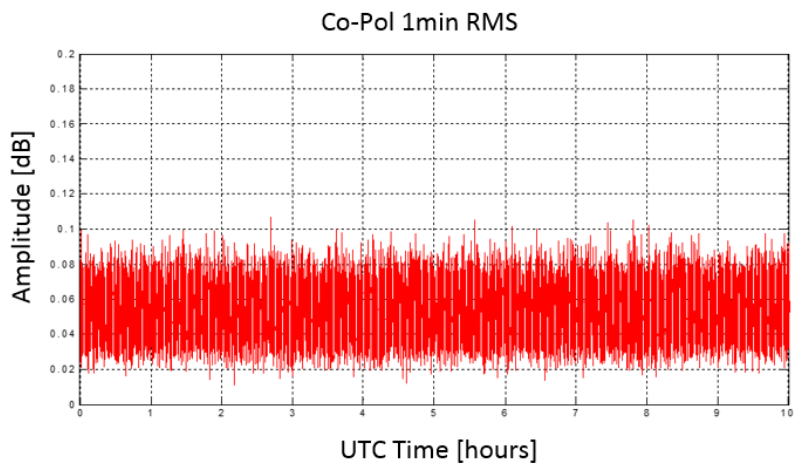

Figure 4: Co-Pol RMS

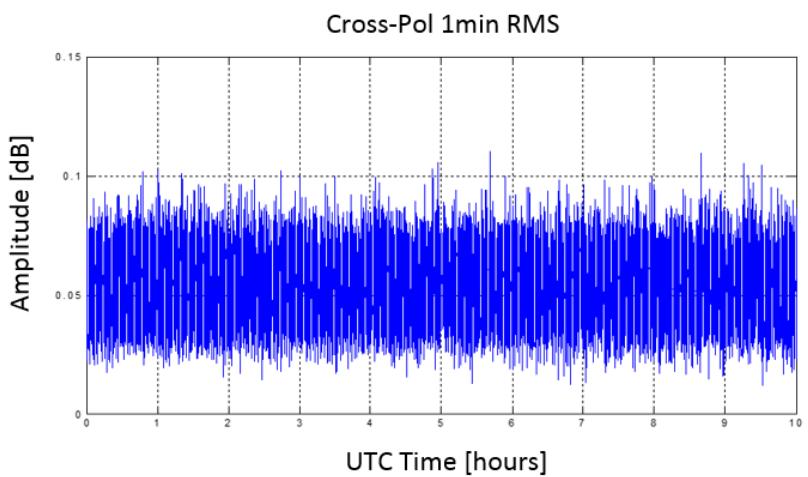

Figure 5: Cross-Pol RMS

\section{OUtDOOR TESTING}

After the indoor laboratory testing was complete the propagation terminal was relocated to the outdoor testing facility on the roof of the laboratory. The satellite ANIK F2 is visible from the outdoor testing facility, which generates a beacon signal of $20.199 \mathrm{GHz}$ only $1 \mathrm{MHz}$ away from the frequency of interest $(20.198 \mathrm{GHz})$. Unfortunately the beacon 
signal from ANIK F2 is linearly polarized and the system is designed to receive the circular polarized signal from THOR 7. This discrepancy results is a in a greatly reduced cross polarization isolation however the results gathered from utilizing ANIK F2 as a source still indicate that the system will meet the specified performance.

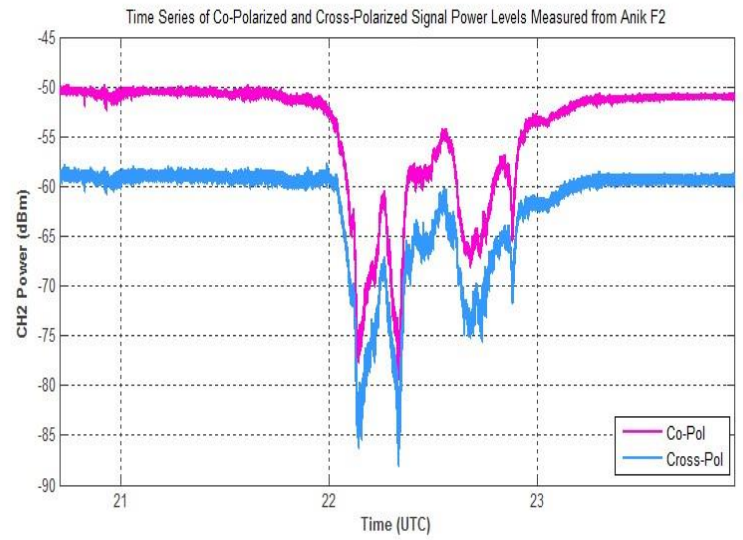

Figure 6: Rain Event

Figure 6 shows the measured co and cross polarized received signals during a rain event. From this data it can be observed that the terminal was able to track the excess attenuation of more than $25 \mathrm{~dB}$ without reaching the noise floor. Figure 7 shows the power spectral density (PSD) of the received signals during the same rain event mentioned in Figure 6 and Figure 8 shows the PSD during clear sky conditions.

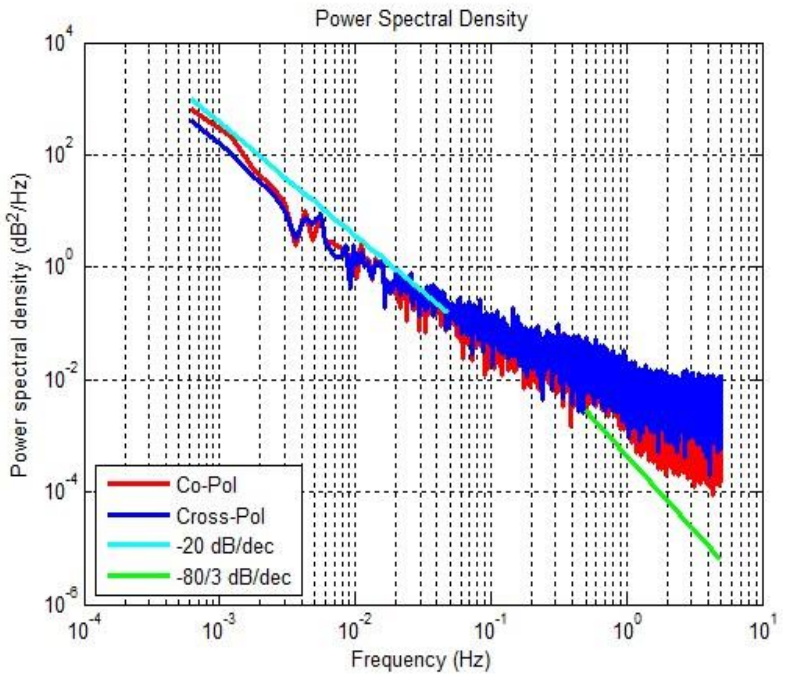

Figure 7: PSD during rain event

The PSD slope of troposphere scintillations and rain are different and allow for the identification of these phenomena within the data. Rain attenuation has a characteristic slope of $20 \mathrm{~dB} /$ decade up to hundreds of hertz [6]. Scintillations however have a characteristic slope of $-80 / 3 \mathrm{~dB} /$ decade $(-8 / 3$ in natural units) [7]. From the figure it can be observed that the unique slopes indicating rain and scintillation are approximately present in the corresponding plots, additionally it shows that the noise floor is reached at approximately $4 \mathrm{~Hz}$ on the co-polarized channel.

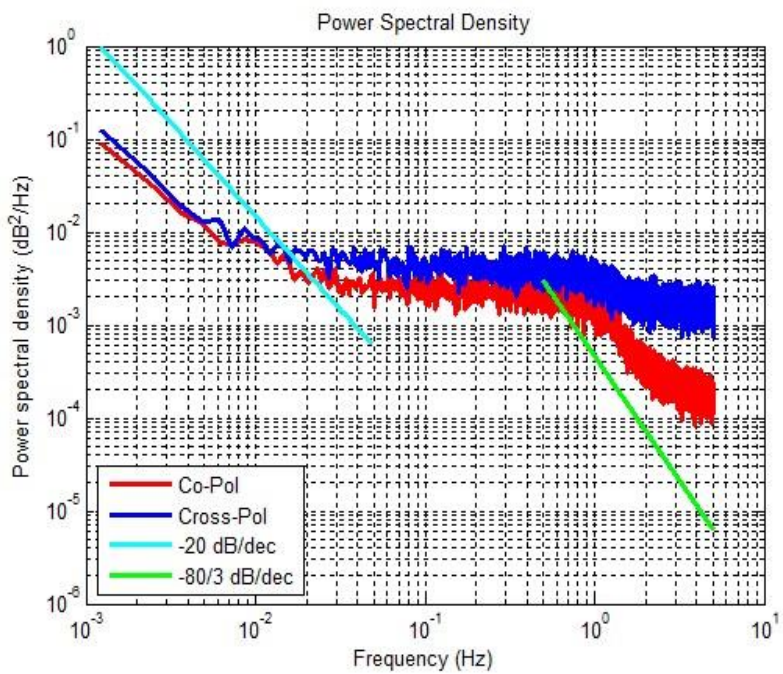

Figure 8: PSD during clear sky

\section{CONCLUSIONS}

The propagation terminal describing in the paper is scheduled to be deployed in Svalbard in the spring of 2016. Based upon the laboratory and outdoor testing completed the system is expected to have a measurement resolution of $.05 \mathrm{~dB}$ on both the co and cross polarized channels and better than $35 \mathrm{~dB}$ of cross polarization isolation. This system will meet or exceed all of the performance requirements and will be used to characterize the atmosphere in the artic region.

\section{REFERENCES}

[1] K. McCarthy, F. Stocklin, B. Geldzahler, D. Friedman, P. Celeste, "NASA's Evolution to Ka-Band Space Communications for Near-Earth Spacecraft," SpaceOps 2010, Huntsville, AL, April 25 - 30, 2010.

[2] J. Nessel, J. Morse, M. Zemba, "Results from Two Years of Ka-band Propagation Characterization at Svalbard, Norway," 8th European Conference on Antennas and Propagation, The Hague, Netherlands, April 2014.

[3] R. Acosta, et. al., "Goldstone Site Test Interferometer," 13th Ka and Broadband Comm. Conference, Turin, Italy, Sep. 2007.

[4] R. Acosta and J. Nessel, "Data Processing for Atmopheric Phase Interferometers", NASA TM 2009-215599

[5] M. Zemba et al., "Frequency Estimator Performance for a SoftwareBased Beacon Receiver," In Proceedings of the 2014 IEEE Antennas and Propagation Conference, July 6-12, 2014, Memphis, TN.

[6] Matricciani, E. (1994), Physical-mathematical model of the dynamics of rain attenuation with application to power spectrum, Electron. Lett., 30, $522-524$.

[7] Tatarski, V. I. (1961), Wave Propagation in a Turbulent Medium,McGraw-Hill, New York.

[8] A. R. Thompson, J. M. Moran, and G. W. Swenson, Interferometry and Synthesis in Radio Astronomy. John Wiley \& Sons, 2001. 